\title{
Phase-field modelling of gravity-capillary waves on a miscible interface ${ }^{\star}$
}

\author{
A. Vorobev ${ }^{1, a}$, A. Ivantsov ${ }^{2}$, and T. Lyubimova ${ }^{2}$ \\ 1 Faculty of Engineering and the Environment, University of Southampton, Southampton, UK \\ 2 Institute of Continuous Media Mechanics Ural Branch RAS, Perm Federal Research Centre, Perm, Russia
}

Received 21 September 2017 and Received in final form 11 October 2017

Published online: 20 November 2017

(C) The Author(s) 2017. This article is published with open access at Springerlink.com

\begin{abstract}
Using the approach of direct numerical simulations we investigate the gravity-capillary waves induced on a horizontal interface between two slowly miscible liquids. It is assumed that the liquids are just brought into contact, and thus the interface is slowly smeared by the action of interfacial diffusion. It is also assumed that the initial shape of the interface is distorted by harmonic perturbations, which results in the development of the gravity-capillary surface waves. The evolution of the binary mixture is modelled on the basis of the phase-field method. Our results show that in the limiting case of negligible diffusion the classical dispersion relations for immiscible interfaces can be reproduced. Although, for the waves with shorter wavelengths such an agreement is more difficult to obtain. The interfacial diffusion brings an additional dissipation to the fluid system, strongly damping the development of the shorter waves. We also show that the mixing (or the transition of a binary system to the state of thermodynamic equilibrium) is intensified by the presence of the surface waves, and this effect is more important when the liquids are slowly miscible, i.e. when the interfacial diffusion is weak.
\end{abstract}

\section{Introduction}

The evolution of hydrodynamic perturbations on the surface of a slowly smearing boundary is studied in order to comprehend the mechanisms of the mixing dynamics of liquid/liquid and gaseous/liquid substances, which is a pre-requirement for numerous chemical reactions, and thus needed for the majority of processes in chemical engineering. Slow miscibility frequently limits the reaction rates and the amounts of the reactants that partake in the chemical process, normally forcing an extensive supply of reactants, and leading to the problems of separation of the products from non-reacted substances. The mixing rate strongly depends on the contact area of the phases, and the surface waves may be expected to intensify the mixing through enlargement of the contact area.

In the current work, the evolution of a disturbed boundary between two miscible liquids is modelled on the bases of the phase-field approach. We consider the initial moments after the contact of two liquids, thus when a binary mixture is in a state different from the state of thermodynamical equilibrium, and when the process of interfacial diffusion occurs across the boundary. Through

\footnotetext{
* Contribution to the Topical Issue "Non-equilibrium processes in multicomponent and multiphase media" edited by Tatyana Lyubimova, Valentina Shevtsova, Fabrizio Croccolo.

a e-mail: A.Vorobev@soton.ac.uk
}

the direct numerical simulations we aim to understand i) the effects of the interfacial diffusion on the dispersion relations of the gravity-capillary waves, and ii) the effect of the surface waves on the intensity of the mixing process of two miscible liquids.

\section{Governing equations: phase-field approach}

The continuum approach for the description of a heterogeneous mixture of two miscible liquids was first proposed by van der Waals [1] and Korteweg [2]. Within the phasefield approach the interface separating two liquids is represented as a transitional boundary of finite thickness. The concentration field $C$, that is defined as the mass fraction of one component in the mixture, is used to trace the evolution of the interfacial boundary.

The specific free energy function for the miscible multiphase system is defined by a function of concentration and gradient of concentration $[3,4]$,

$$
f(C, \nabla C)=f_{0}(C)+\frac{\epsilon}{2}(\nabla C)^{2} .
$$

In this expression, the second term takes into account the dynamic surface tension effects at a miscible interface. The capillary constant $\epsilon$ is assumed to be sufficiently small so to be able to neglect this term everywhere except at 
the places of large concentration gradients, i.e. where the interface is located.

The double-well potential is frequently used for the classical part of the free energy function, $f_{0}$, especially, when the primary interest is tracing the evolution of the immiscible interface [5]. We however want to consider the interface between two miscible liquids. In [6], it was proposed to determine $f_{0}$ on the basis of the Landau formula,

$$
f_{0}(C)=a\left(C-C_{c r}\right)^{2}+b\left(C-C_{c r}\right)^{4}
$$

This expression was originally proposed for description of the states of the binary mixtures near the thermodynamic critical point. In particular, it should well represent the equilibrium states of the binary mixture with the so-called upper critical temperature (consolute point), when the mixture is homogeneous in equilibrium above the critical temperature and may be heterogeneous below the critical temperature. For convenience, we will shift the reference point for the concentration field as $C \rightarrow\left(C-C_{c r}\right)$.

The parameters $a$ and $b$ are treated as two phenomenological coefficients with the values taken so that to provide better matching with the experimental data. We will however assume that $b$ remains always positive, while $a$ can be either positive or negative defining whether the system is single and two phase in equilibrium.

The free energy function (1) can be used to re-derive the Navier-Stokes equations for the mixture, that was done by Lowengrub and Truskinovsky [7]. The so-obtained full Cahn-Hilliard-Navier-Stokes equations are however very complex for the numerical treatment because of quasi-compressibility (dependence of the mixture density on concentration) that forces to use the full continuity equation even for the description of two incompressible liquids. The Boussinesq approximation of the full equations, derived in [6], is used in the current work to define the evolution of the gravity-capillary waves on a surface of two miscible liquids.

The governing equations reflect the conservation of momentum, species, and mass

$$
\begin{aligned}
& \frac{\partial \boldsymbol{u}}{\partial t}+(\boldsymbol{u} \cdot \nabla) \boldsymbol{u}=-\nabla \Pi+\frac{1}{R e} \nabla^{2} \boldsymbol{u}-C a \nabla^{2} \mu+2 G r C, \\
& \frac{\partial C}{\partial t}+(\boldsymbol{u} \cdot \nabla) C=\frac{1}{P e} \nabla^{2} \mu \\
& \nabla \cdot \boldsymbol{u}=0 \\
& \mu=G r(\boldsymbol{r} \cdot \gamma)+2 A C+4 C^{3}-C a \nabla^{2} C .
\end{aligned}
$$

Here, the standard notations are used for the variables. These equations are applied to the whole multiphase system, including the interface. One sees that the NavierStokes equation contains an additional force that defines the morphology of the interface. The diffusion is defined by the generalized Fick's law, i.e. through the gradient of the chemical potential $\mu$. The diffusion term includes in addition to the usual concentration diffusion, the barodiffusion terms. $\gamma$ is the unit vector directed upward.

The equations are written in non-dimensional form and include the following non-dimensional parameters. The
Grashof number,

$$
G r=\phi \frac{g L_{*}}{\mu_{*}},
$$

the Reynolds number,

$$
R e=\frac{\rho_{*} \mu_{*}^{1 / 2} L_{*}}{\eta_{*}},
$$

the Peclet number,

$$
P e=\frac{\rho_{*} L_{*}}{\alpha \mu_{*}^{1 / 2}},
$$

the capillary number,

$$
C a=\frac{\epsilon}{\mu_{*} L_{*}^{2}} .
$$

In these formulae $L_{*}$ is the typical size, $\rho_{1}$ is used as the density scale, $\mu_{*}$ is the unit of the chemical potential, $\eta_{1}$ is the viscosity scale, $\alpha$ is the mobility constant, and $\phi=$ $\frac{\rho_{2}-\rho_{1}}{\rho_{1}}$ is the density contrast, with $\rho_{2}$ and $\rho_{1}$ being the densities of the pure components of the binary mixture.

The parameter $A=a / b$ is used to define the thermodynamic state of the mixture.

It is necessary to note that the non-dimensional parameters are different from the classical parameters, that would be defined on the basis of the standard set of phenomenological parameters such as the surface tension and diffusion coefficients. We use the common names for these parameters, as they appear in front of the corresponding terms in the governing equations and generally have the meanings similar to those associated with the classical non-dimensional numbers. $C a$ is called here the capillary number, however, in some other works (e.g., [7]), the same parameter is called the Cahn number.

It is also necessary to note that the governing equations (3) were derived for two liquids with different viscosities. The difference in viscosities was, however, assumed to be small, and only one single Reynolds number is used to characterize the binary mixture. For the case when the viscosity difference is large, the governing equations need to be reconsidered, bringing several new terms to the equations. This has not been done in the present work.

The surface tension coefficient associated with the phase boundary can be determined as

$$
\sigma=C a \int_{-\infty}^{\infty}\left(\frac{\mathrm{d} C}{\mathrm{~d} x}\right)^{2} \mathrm{~d} x
$$

where $x$ is the coordinate across the interface. For a heterogeneous binary mixture in the state of thermodynamic equilibrium and with no gravity effect with a flat interface, the thickness of the interface can be evaluated as $\delta_{0}=\sqrt{-C a / A}$, and the surface tension coefficient as $\sigma_{0}=\frac{1}{3} \sqrt{-C a A}$

On macroscopic scales, the interface between two liquids is infinitely sharp, and the limit of $\delta \rightarrow 0$ is of the primary interest for the phase-field approach. As shown in [8], the limit of the sharp interface is not easy to obtain, 


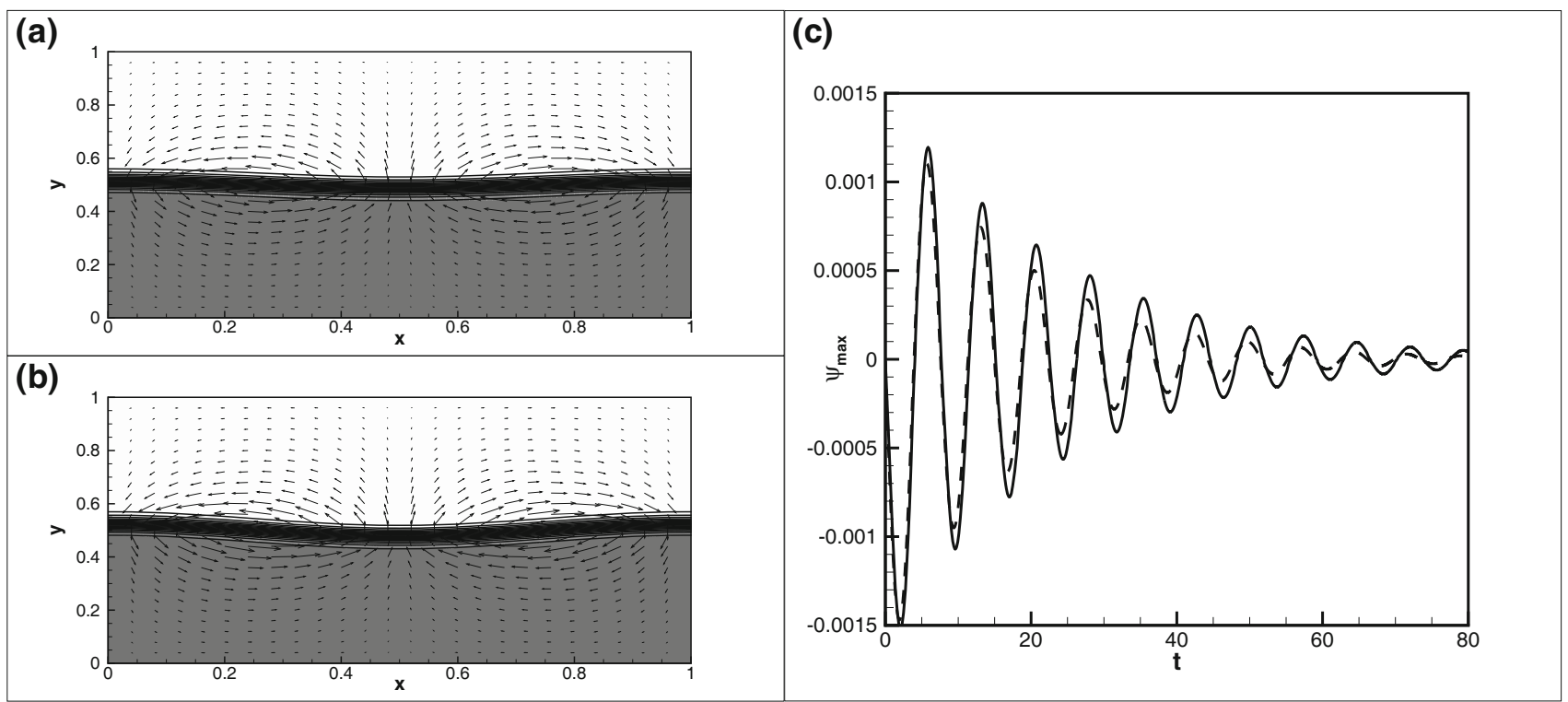

Fig. 1. (a), (b) The fields of concentration and velocity at $t=20$. Panel (a) shows the data obtained with the grids of $200 \times 200$ grid nodes and panel (b) shows the data obtained with $400 \times 400$ grid nodes. (c) The maximum value of the field of streamfunction vs. time (solid line for the run with $400 \times 400$ grid nodes and dashed line for the run with $200 \times 200$ grid nodes). The shown data are obtained at $A=-0.5, G r=-0.1, P e=10^{5}, R e=2000, C a=4 \cdot 10^{-4}, \delta=0.0283$.

as both the interface thickness and the surface tension coefficients depend on $C a$, and both values would decrease if $\mathrm{Ca}$ tends to zero. Hence, a single decrease in the values of the capillary number would make the interface thinner, but a thinner interface would be also endowed with smaller surface tension.

\section{Problem statement and numerical approach}

It is assumed that two pure liquids of different densities are brought into contact, so that the heavier liquid is placed at the bottom (i.e., $G r<0$ ). The initial shape of the interface is given by the following concentration profile:

$$
C=\frac{1}{2} \tanh \left[\frac{y-\left(\frac{1}{2}+a \cos (k x)\right)}{\delta}\right] .
$$

The pure liquids are defined by the concentration levels, $\pm 1 / 2$. The waves on the interface are defined by the amplitude $a$ and the wave number $k=2 \pi / \lambda$ (here $\lambda$ is the wavelength). The thickness of the interface $\delta$ is treated as an additional independent parameter of the model. For the computations reported below, the amplitude of perturbations was fixed $a=0.1$; the time evolution perturbations of different wavelength and the interfacial boundaries of different initial thicknesses $(\delta \ll \lambda)$ were examined.

The computational domain is represented by a rectangular region with periodic boundary conditions at the left and right sides and with the rigid walls at the top and bottom boundaries (the Cartesian coordinates $x$ and $y$ are used for the horizontal and vertical dimensions). Namely, the following boundary conditions are imposed at the lower and upper boundaries of the domain:

$$
y=0,1: \quad u_{x}=u_{y}=0, \quad \frac{\partial \mu}{\partial x}=\frac{\partial C}{\partial x}=0 .
$$

Thus, the lower and upper boundaries are assumed to be impermeable. The no-slip boundary conditions are used for the velocity field. For the chemical potential, we impose the absence of the diffusive flux through these boundaries. The conditions for the concentration should reflect the wetting conditions. We consider the simplest case when the molecules of the mixture components interact with the wall equally, so the contact angle is $90^{\circ}$. In fact, the contact line never reaches either the top or bottom boundaries, so the wetting properties are not important for the current study.

The height of the domain is used as the length scale. The horizontal size of the domain is equal to the wavelength of the induced perturbation, and hence is different for different runs.

For the numerical solution, the equations were rewritten into the streamfunction $(\psi)$-vorticity $(\Omega)$ formulation, defined by the following relations:

$$
\Omega=\frac{\partial u_{y}}{\partial x}-\frac{\partial u_{x}}{\partial y} ; \quad u_{x}=\frac{\partial \psi}{\partial y}, \quad u_{y}=-\frac{\partial \psi}{\partial x} .
$$

The resultant equations are solved using the finitedifference method on a uniform mesh. The explicit first order in time and second order in space discretization scheme was used.

\section{Results}

The typical snapshots of the fields of concentration and velocity are depicted in figs. 1(a), (b). In these figures, one sees the sharp interfaces that separate two phases and the flow vortices that penetrate through the interfaces. Figure 1(c) shows the time evolution of the maximum value 


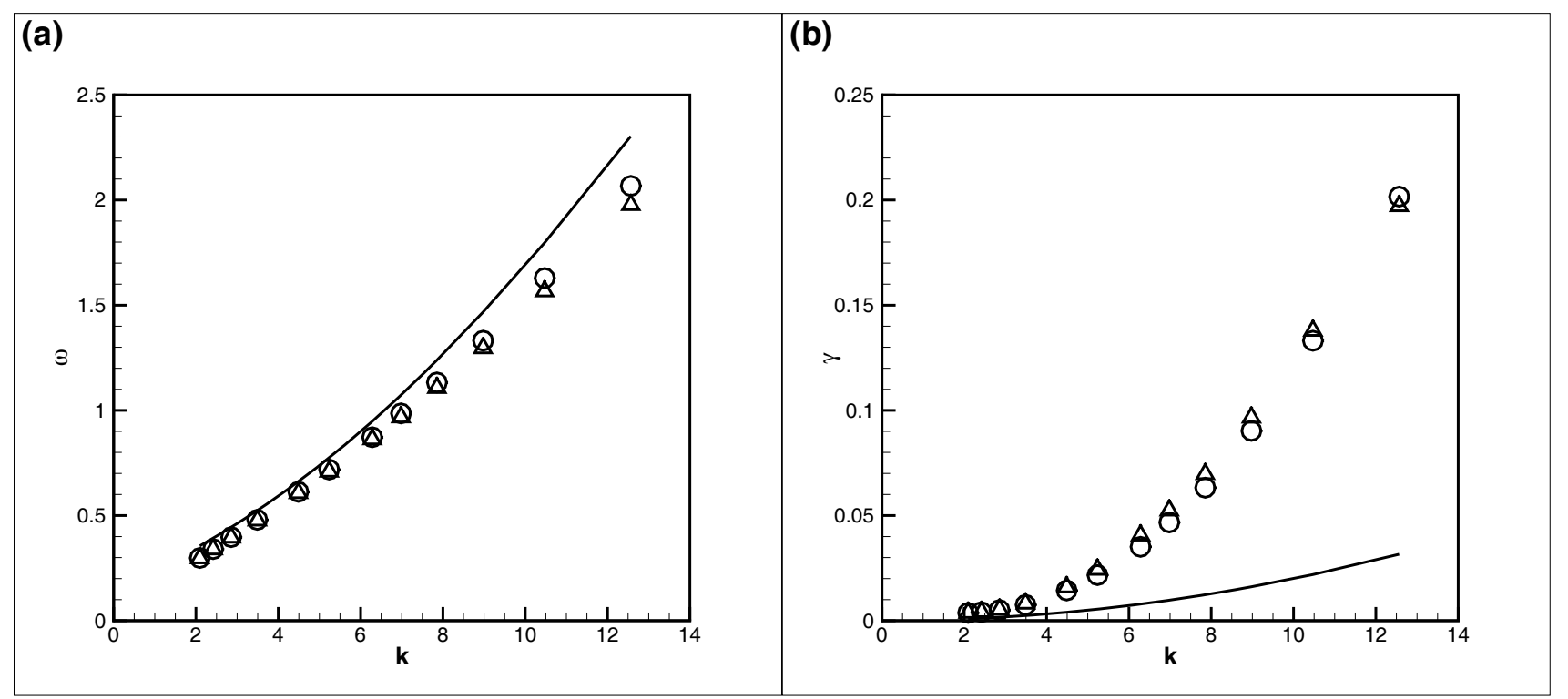

Fig. 2. The frequency (a) and the decay rate (b) of oscillations $v s$. wave number. The data are shown for $A=-0.5, G r=0.1$, $C a=4 \times 10^{-4}, R e=10000, \delta=0.0283$, and for $P e=10^{5}$ (triangles) and $P e=10^{6}$ (circles). The solid line depicts the theoretical curves (15) for an immiscible infinitely thin interface.

of the streamfunction, that demonstrates a decaying oscillatory behavior. These time dependencies are further used to obtain the frequency of oscillations, $\omega$, and the rate of decay, $\gamma$. The data in fig. 1 are shown for two different grids, with 200 and 400 grid nodes in the vertical direction (the size of the computational domain in the horizontal direction is adjusted to the wavelength, but the size of the grid cell remains the same). There are just tiny differences in the data obtained with the use of these two grids, which, first, illustrates the grid independence of our results and, second, confirms that the data obtained with the help of the rough grid (200 grid nodes) are sufficiently reliable (the frequencies obtained with these two grids are 0.861 and 0.859 and the decay rates are 0.0561 and 0.0440). However, all the results shown below (except for fig. 2) are obtained using the grids with 400 points in the vertical direction.

Next, we aim to investigate the dispersion relations for the gravity-capillary waves at different values of the Peclet number. At higher Peclet numbers the interface between two fluids should behave similarly to the immiscible case, so the dispersion relations should become sufficiently close to the classical relations

$$
\omega^{2}=\frac{1}{2} G r k+\frac{\sigma}{2} k^{3}, \quad \gamma=\frac{2}{R e} k^{2} .
$$

These formulas are valid for the weakly viscous case. Here, $\sigma=C a /(3 \delta)$ is the surface tension coefficient (11) calculated for the initial state (12).

Figure 2 depicts the dispersion relations for a binary mixture at higher Peclet numbers. In this case, the calculated curves for the frequency of the waves lie very close to the curve calculated from the theoretical formula (15). Although, the decay of the waves occurs faster compared to the predictions (15), especially for the waves with shorter wavelengths, which could be explained by the fact that in our calculations the interface is of the finite thickness.

The finite thickness of the interface introduced within the phase-field approach is an artificial parameter, as the thickness of a real interface is just several molecular layers, i.e. zero for the macroscopic theory. Figure 3 shows the behavior of the binary mixture for different values of the parameter $\delta$. The results demonstrate that in the limit of longer wavelengths, the dependence on the interface thickness is rather weak, and the data obtained for three different values of $\delta$ almost coincide. For the shorter waves the difference in the data is getting more significant. It is also interesting, that the gradual decrease of the interface thickness makes the difference of the calculated frequency and decay rate even more different from the theoretical curve (15).

The classical dispersion relation (15) says that the capillary effects are weaker for longer waves, and these effects get much stronger for shorter waves. For longer waves, the limiting behavior of a sharp interface can be obtained by a gradually decrease of the interface thickness. This however is not working for shorter waves, when the capillarity effects are essential.

The coefficient of surface tension is given as the ratio of the capillary number over the interface thickness. If the interface thickness is changed at the same capillary number, then at least the initial value of the surface tension coefficient is different. For the runs with the fixed value of the surface tension coefficient, we need to simultaneously change the capillary number and the interface thickness, so keeping their ratio unmodified. The results of these calculations are shown in fig. 4. These results demonstrate much better convergence to the limiting behaviour of an infinitely thin interface boundary. Although, again for shorter wavelengths the effects of diffusion and 
(a)

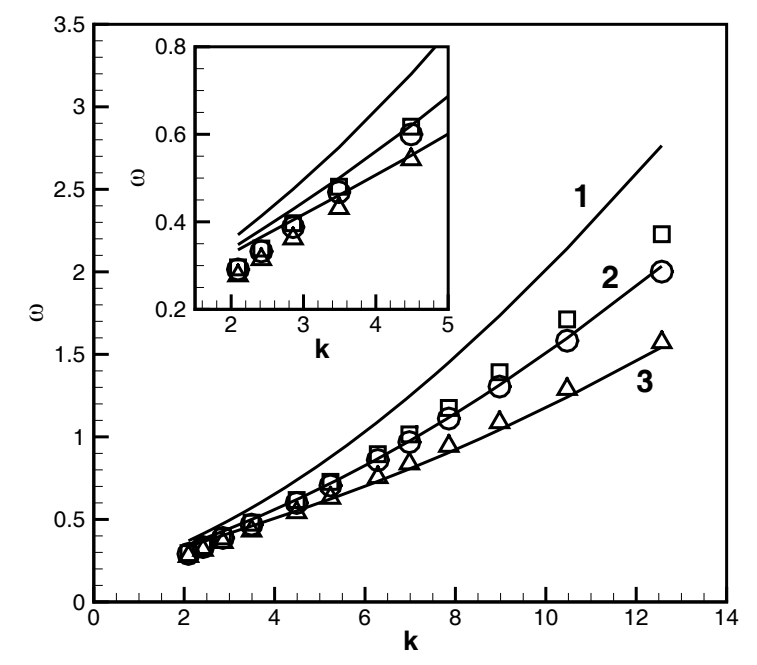

(b)

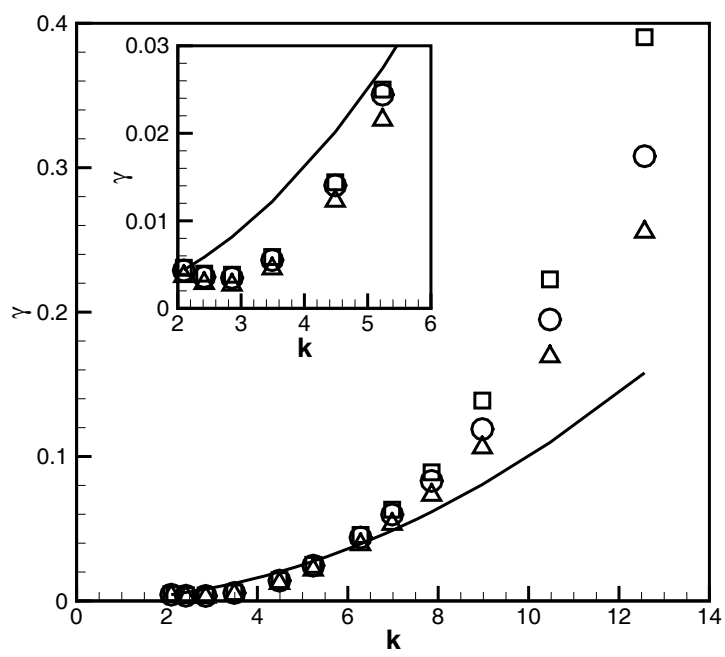

Fig. 3. The frequency (a) and the decay rate (b) of oscillations $v s$. wave number. The data are shown for $A=-0.5, G r=0.1$, $P e=10^{5}, C a=4 \times 10^{-4}, R e=2000$, and $\delta=0.0141$ (squares), $\delta=0.0283$ (circles), and $\delta=0.0566$ (triangles). The solid lines depict the theoretical data (15) for the cases of (1) $\delta=0.0141,(2) \delta=0.0283$, (3) $\delta=0.0566$ in (a) and the theoretical data for $\gamma$ for $R e=2000$ in (b).

(a)

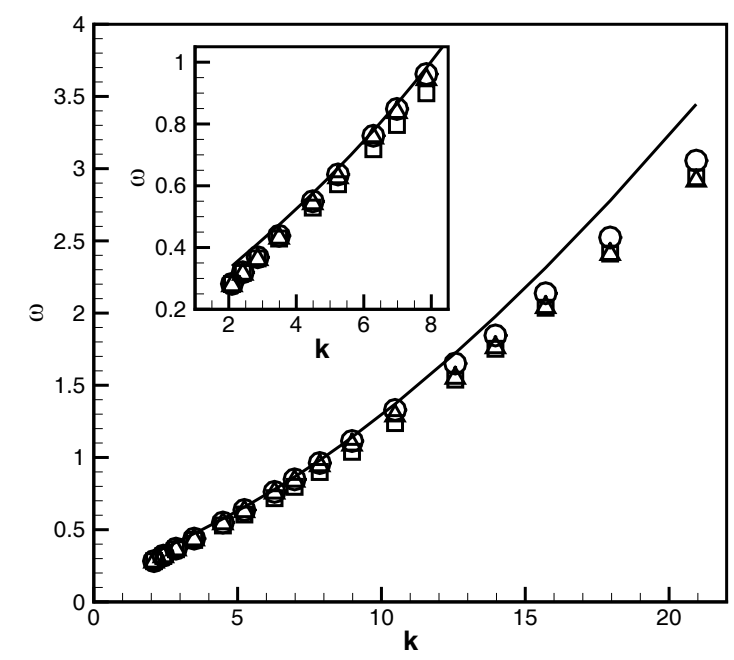

(b)

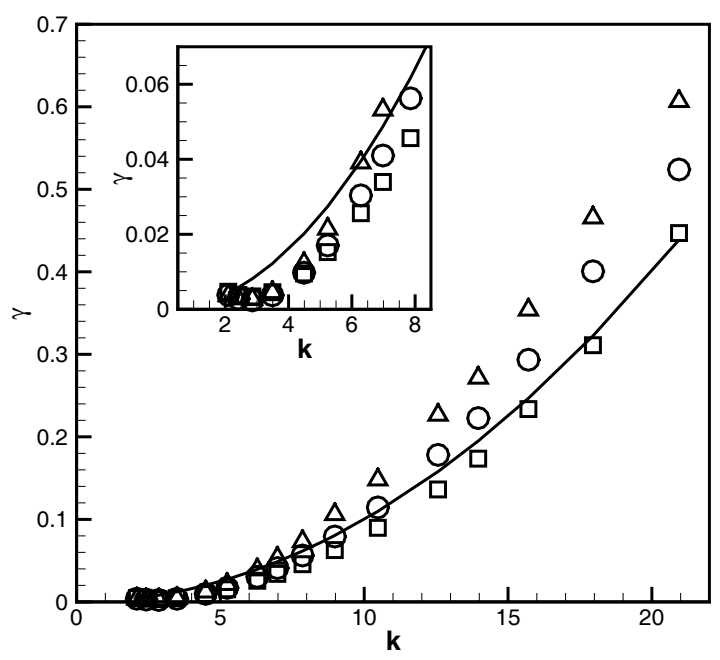

Fig. 4. The frequency (a) and the decay rate (b) of oscillations vs. wave number. The data are shown for $A=-0.5, G r=0.1$, $P e=10^{5}, R e=2000$, and for different values of capillary number and interface thickness, $C a=10^{-4}, \delta=0.0141$ (squares), $C a=2 \times 10^{-4}, \delta=0.0283$ (circles), and $C a=4 \times 10^{-4}, \delta=0.0566$ (triangles). The solid line depicts the theoretical curve (15).

capillarity are stronger, and the difference between the obtained results becomes also stronger in this limit.

It should be also noted that the curves in fig. 2 are plotted for $R e=10000$, and the curves in figs. 3 and 4 are plotted for $R e=2000$. All calculated data lie sufficiently close to the theoretical data, although at $R e=10000$ the decay rate is always above the theoretical predictions, while at $R e=2000$ the decay rate is lower than the theoretical predictions for longer wavelengths, and the data lie above the theoretical curve for the shorter wavelengths.

Finally, we also consider how the waves induced at the interface affect the rates of smearing of interfacial bound- aries. For the current 2D study, the interface thickness was calculated as an area, where $|C|<0.45$, divided by the interface length. Although, as we only focused on small amplitude oscillations, the interface length was taken as a constant equal to the length of an unperturbed (flat) interface.

In fig. 5 the time changes of the interface thickness are plotted for the different Peclet numbers that define the intensity of the interfacial diffusion. At lower Peclet numbers the diffusion is stronger, and, obviously, the interface thickness grows faster. One can also notice that the surface waves intensify the smearing, as was expected. Although, 

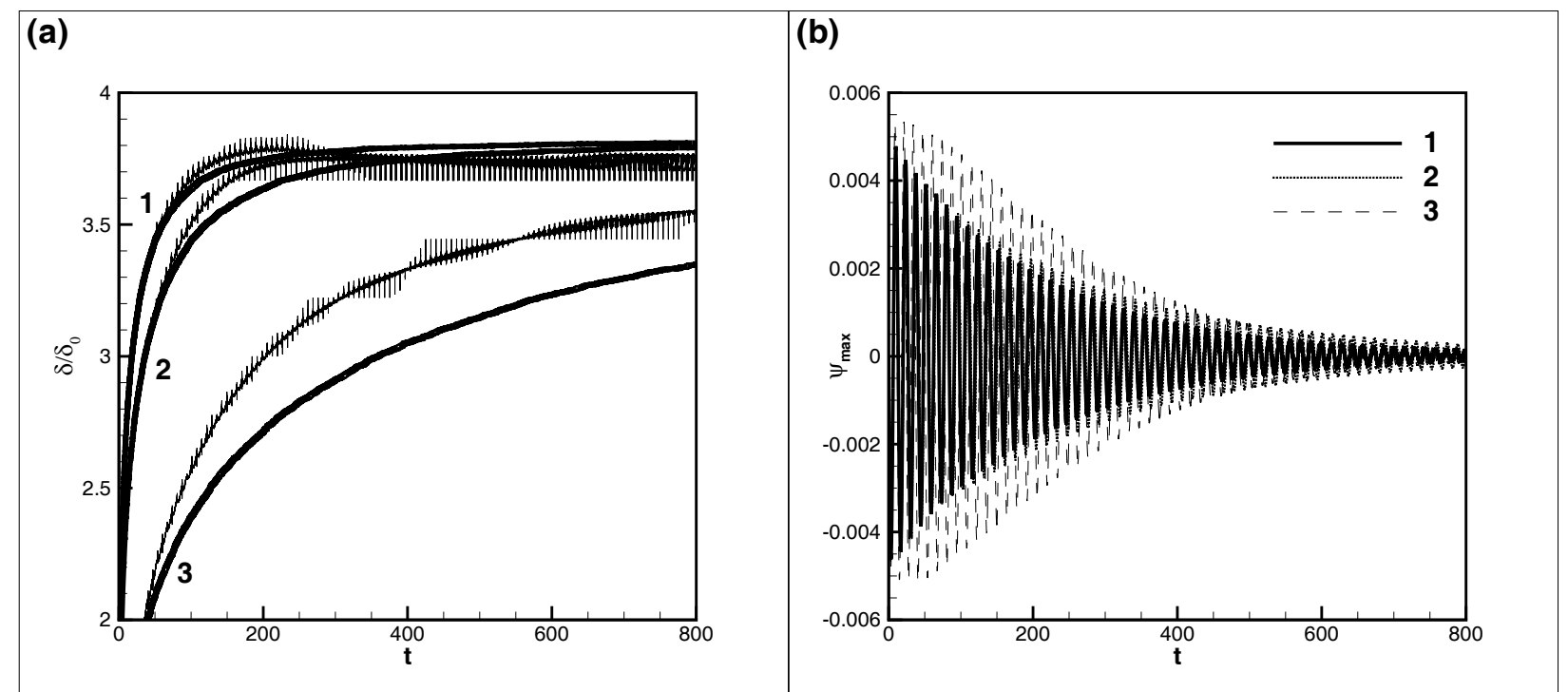

Fig. 5. (a) The time evolution of the interface thickness for the cases without (bold lines) and with (thin oscillating lines) the imposed surface waves. (b) The time evolutions of the streamfunction for the calculations with the imposed surface waves. Here $\delta_{0}=0.0071$ is the interface thickness at the initial time moment. The calculations are performed for $R e=10^{4}, A=-0.5$, $G r=0.1, k=3.14:(1) P e=5 \times 10^{4} ;(2) P e=10^{5}$; (3) $P e=10^{6}$.

for lower Peclet numbers (cases 1 and 2), the effect of the waves is weak, so the time needed for the system to attain the state of thermodynamic equilibrium remain virtually unchanged. At higher Peclet numbers (case 3), the enhancement of the mixing process by the surface waves is stronger, so the curves that depict the transition of the binary mixture to the state of thermodynamic equilibrium become substantially different for the runs with initially flat and disturbed boundaries.

\section{Conclusions}

In the current work we aimed to assess whether the phasefield approach could accurately reproduce the dynamics of an interface between two miscible liquids that are brought into contact. We assume that at the time of the contact, the binary mixture is thermodynamically non-equilibrium, and equilibration is primarily driven by the interfacial diffusion, a very slow process. Traditionally, it is assumed that the surface waves increase the contact area of two liquids, and hence should enhance the mixing, or the transition of the binary mixture to the state of thermodynamic equilibrium. Thus, the second aim of the work was to assess the capability of phase-field approach to capture the dynamics of the waves propagating on a slowly miscible interface, and, finally, to understand whether the mixing dynamics can be controlled by the surface waves.

The results of our study show that the overall dynamics of the miscible boundary could be correctly reproduced with the help of phase-field boundary. The boundary remains sharp, and by variation of the values of the Peclet and Reynolds number, the typical time scales for the diffusive and viscous processes can be separately set. At very high Peclet numbers, the interfacial diffusion is negligible, and at sufficiently high Reynolds numbers the gravitycapillary waves could be induced at the boundary. For the waves with longer wavelengths, we managed to obtain a good agreement with the classical relationships. The shorter waves on a miscible interface, are characterized by much stronger decay rates, due to additional diffusive damping. We also showed that the surface waves do also affect the diffusion process, especially at higher Peclet numbers, when diffusion is weaker, clearly intensifying the smearing of the interfacial boundary, and thus increasing the rate of transition to the state of thermodynamic equilibrium.

The financial support of the Royal Society (Ref. IE160277), and the Russian Foundation for Basic Research (Grant No. 165110079), and the Government of Perm region, Russia (Contract No. C-26/004.03) is gratefully acknowledged.

\section{Author contribution statement}

AV, AI and TL contributed to the design and implementation of the research, to the analysis of the results and to the writing of the manuscript.

\section{Availability of data and materials}

All data supporting this study is openly available from the University of Southampton repository at https://doi.org/10.5258/SOTON/D0257.

Open Access This is an open access article distributed under the terms of the Creative Commons Attribution License (http://creativecommons.org/licenses/by/4.0), which permits unrestricted use, distribution, and reproduction in any medium, provided the original work is properly cited. 


\section{References}

1. J.S. Rowlinson, J. Stat. Phys. 20, 197 (1979).

2. D.J. Korteweg, Arch. Neérl. Sci. Exactes Nat. Sér. II 6, 1 (1901).

3. Ya.B. Zeldovich, Zh. Fiz. Khim. 23, 931 (1949).
4. J.W. Cahn, J.E. Hilliard, J. Chem. Phys. 28, 258 (1958).

5. J. Jacqmin, J. Comput. Phys. 155, 96 (1999).

6. A. Vorobev, Phys. Rev. E 82, 056312 (2010).

7. J. Lowengrub, L. Truskinovsky, Proc. R. Soc. A 454, 2617 (1998).

8. A. Kheniene, A. Vorobev, Phys. Rev. E 88, 022404 (2013). 